\title{
Estereología Renal en el Cobayo (Cavia porcellus)
}

\author{
Renal Stereology in the Guinea Pig (Cavia porcellus)
}

*José Luis Zapata Barrera; "Mariano del Sol \& **Bélgica Vásquez

ZAPATA, B. J. L.; DEL SOL, M. \& VÁSQUEZ, B. Estereología renal en el cobayo. Int. J. Morphol., 27(2):419-424, 2009.

RESUMEN: El cobayo (Cavia porcellus) es un roedor perteneciente al Orden Rodentia y a la Familia Caviidae, utilizado como animal de laboratorio y de consumo humano. Los parámetros cuantitativos del riñón entregan importante información de su morfofunción dada su labor en la homeostasis del organismo. El objetivo de este estudio fue describir el riñón de cobayo (Cavia porcellus), analizando las características estereológicas para futuros estudios experimentales. Se utilizaron 5 cobayos machos, obtenidos del Bioterio de la Universidad de La Frontera, Temuco, Chile. El riñón de cobayo pesó 3,2 g, aproximadamente. El riñón posee 140.298 glomérulos en total, $\mathrm{Nv}$ de $458 \mathrm{~mm}^{3}$, Vv de 7,89\% y Sv de 3,58 $\mathrm{mm}^{2} /$ $\mathrm{mm}^{3}$. El volumen glomerular del riñón fue de $1,73 \times 10^{-4} \mathrm{~mm}^{3}$ y el diámetro glomerular de $90 \mu \mathrm{m}$. Factores como especie, edad, peso corporal, peso y volumen renal, son importantes a considerar, ya que diferencian los resultados en investigaciones morfofuncionales.

PALABRAS CLAVE: Riñón; Cavia porcellus; Estereología.

\section{INTRODUCCIÓN}

El riñón es un órgano que realiza una amplia variedad de funciones para mantener la homeostasis, siendo su unidad funcional la nefrona. Tanto en condiciones normales como patológicas, es posible detectar modificaciones ya sean macro o microscópicas, cambios que pueden ser cuantificados a través de estudios estereológicos.

El cobayo, guinea pig, Cavia porcellus fue descrito por primera vez por Linnaeus (1758) como Mus porcellus. Es un roedor perteneciente al Orden Rodentia, Familia Caviidae, originario de la zona andina de Sudamérica, utilizado como prototipo de modelo de laboratorio desde el siglo XIX en Europa y es un importante alimento en los países andinos. Según Spotorno et al. (2004) este animal tiene una alta relación filogenética con Cavia tschudii un roedor silvestre de Perú y Chile.

Existen en la literatura algunos estudios sobre las características anatómicas renales de los cobayos. Martín et al. (2004) estudiaron la anatomía renal del cobayo doméstico (Cavia aperea), señalando que éstos tienen la forma de judía, de superficie lisa y de color rojo obscuro.

\footnotetext{
* Facultad de Medicina, Universidad de La Frontera, Temuco, Chile.

** Universidad Autónoma de Chile, Temuco, Chile.
}

Fawcett (1995) señaló que la médula renal humana está subdividida en 6 a 10 pirámides renales y sus límites laterales quedan definidos por las columnas renales a nivel de corteza. Un lóbulo renal está constituido por una pirámide y el tejido cortical que se le superpone. Por lo cual, cada lóbulo del riñón multilobulado del Hombre se corresponde a una unidad completa unipiramidal de los riñones unilobulados de los roedores de laboratorio utilizados para experimentación.

Según Frandson \& Surgeon (1995) el riñón humano contiene aproximadamente 1 millón de nefronas, de las cuales sólo una parte funcionará en condiciones normales. El diámetro del glomérulo humano varía de 150 a $200 \mu \mathrm{m}$ (Mandarim-de-Lacerda, 2000), en el caballo el corpúsculo renal mide $220 \mu \mathrm{m}$ de diámetro y en el gato $120 \mu \mathrm{m}$ (Henrikson, 1993).

De acuerdo a lo señalado por Cullen-McEwen et al (2003), un bajo número de nefronas heredado o adquirido se ha relacionado con un mayor riesgo de desarrollo de hipertensión e insuficiencia renal. Un peso bajo al nacer resul- 
ta en una disminución del número de glomérulos y más aún, predispone al desarrollo de hipertensión en la vida adulta (Mañalich et al., 2000).

Existen algunos datos estereológicos sobre el número de glomérulos normales en riñones de diversas especies, como ratones, diferentes cepas de ratas, conejos, perros, cerdos, ovejas, monos y humanos. También existen estudios experimentales en riñones de animales en los cuales se determinó además del número de glomérulos, el volumen glomerular, entre otros parámetros estereológicos.

Como lo señalara Nyengaard (1999), el número, tamaño y distribución de las nefronas, células y otros componentes contienen información importante acerca de la función del riñón. Por lo tanto, es fundamental que varios de estos componentes sean medidos correctamente. La cuantificación de estos componentes es también relevante cuando se analizan los riñones frente a trauma, químicos y enfermedades.

El objetivo de este estudio fue determinar algunos parámetros estereológicos del riñón del cobayo (Cavia porcellus) con la finalidad de sentar las bases de futuros estudios experimentales, permitiéndonos detectar las eventuales modificaciones estructurales sufridas por este órgano en diferentes condiciones.

\section{MATERIAL Y MÉTODO}

Se utilizaron 5 ejemplares de cobayos machos ( $\mathrm{Ca}$ via porcellus) adultos, sanos, mantenidos en cautiverio y alimentados con pellets comercial, zanahorias y frutas ad limitum, en el Bioterios de la Facultad de Medicina de la Universidad de La Frontera, Temuco, Chile.

Estos animales fueron sacrificados por traumatismo encéfalo craneano. Posteriormente se determinaron sus peso y talla cérvico caudal. Para la descripción anatómica y mesoscópica in situ de los riñones se utilizó material quirúrgico ad hoc y una lupa Ransor (10X).

Los 10 riñones ( 5 derechos y 5 izquierdos), fueron pesados utilizando una balanza analítica marca Chyo JK 180, (precisión 0,01g). Se determinó la longitud céfalocaudal, latero-lateral y dorso-ventral utilizando un paquímetro digital marca Scarrett (ME-6“/150mm). El volumen renal fue obtenido a través del método de Sherle (1970). Luego los riñones fueron fijados en formalina tamponada $10 \%$, por 24 horas. De cada riñón se obtuvie- ron 5 trozos, los cuales fueron cortados utilizando el método Orientator descrito por Mattfeldt et al. (1990). Los trozo de cada riñón fuero procesados según protocolos de deshidratación, aclaración e inclusión en Paraplast Tissue Prep ${ }^{\circledR}$ marca Fisher Scientific. La orientación de cada trozo en la confección de los tacos se realizó al azar.

Para la cuantificación de las estructuras renales se utilizó el método de Disector, descrito por Stereo (1984) y un sistema test multipropósito M42 descrito por Weibel et al. (1966), para lo cual fue necesario determinar el volumen del test, que corresponde al área del test M42 calibrado microscópicamente y multiplicado por el espesor del Disector. Según Mandarim-de-Lacerda (2000) el espesor ideal del Disector debe ser cerca de 1/3 del diámetro de la estructura que se quiere estudiar. Así, para determinar el diámetro medio del glomérulo del cobayo se realizó una adaptación de la técnica de reconstrucción anatómica descrita por Mandarim de Lacerda (1995). Para ello, se seleccionó un taco al azar y se realizaron cortes consecutivos de $5 \mu \mathrm{m}$ de espesor, los cuales fueron teñidos con H.E. Luego se tomaron fotos de la misma área en el seriado, a través de una lupa Olympus ${ }^{\circledR}$ con una cámara fotográfica digital marca Samsung ${ }^{\circledR}$ modelo Digimax A 403 de 4,1 megapíxeles, en formato JPEG. Las fotos que fueron observadas en el visor de imágenes y fax Windows. Se estudiaron 20 glomérulos, siguiendo y cuantificando las imágenes en que se podía observar un mismo glomérulo. Al multiplicar el número de imágenes por el espesor de los cortes histológicos se obtuvo el diámetro medio glomerular que corresponde a $90 \pm 9,32 \mu \mathrm{m}$.

Los cortes histológicos para este estudio se realizaron con un micrótomo rotatorio marca Microm ${ }^{\circledR}$ HM335 E, con un grosor de $5 \mu \mathrm{m}$, tomando un corte cada $30 \mathrm{~mm}$, dos por taco. En total, se obtuvieron 100 cortes histológicos, 10 por cada riñón. Luego fueron montados en portaobjetos y teñidos con H.E.

Para el análisis estereológico se tomaron fotografías en formato JPEG de los cortes histológicos y de una barra calibradora marca Carl Zeiss ${ }^{\circledR}$ modelo AXIOSKOP 40 (objetivo 10X), con cámara incorporada marca Canon ${ }^{\circledR}$ modelo Power Shot G6 de 7,1 megapíxeles y software Capturador Zoom Browser EX.

Para realizar el método Disector, se analizó un mismo campo microscópico de los dos cortes histológicos obtenidos en paralelo con $30 \mu \mathrm{m}$ de separación en cada taco. Luego, se analizaron 5 campos por trozo histológico, para lo cual se capturaron 5 fotografías de 5 campos distintos seleccionados en forma aleatoria en el corte histológico superior y 5 fotos de los mismos 5 campos en 
el corte histológico inferior, resultando 500 campos en total.

Posteriormente, las imágenes fueron proyectadas en una pantalla plana de LCD de 15,4 pulgadas de un notebook marca Acer ${ }^{\circledR}$ modelo Aspire 5610z, con el software de galería fotográfica de Windows XP. Sobre la pantalla se colocó el sistema test M42.

Los parámetros estereológicos medidos en el glomérulo de cobayo fueron: densidad de número $(\mathrm{Nv})$, densidad de volumen $(\mathrm{Vv})$, densidad de superficie $(\mathrm{Sv})$. Además, se calculron el promedio $\mathrm{X}$, la desviación estándar (D.E.), el error estándar (E.E.: D.E./ $\sqrt{ }$ n) (n: número de especies estudiadas) y el coeficiente de variación (C.V.: E.E./X). Se consideró un índice de significancia menor o igual a 0,05 p con probabilidad de un $95 \%$.

\section{RESULTADOS}

El peso promedio de los 5 cobayos fue de $711 \mathrm{~g}$ (D.E. 212,1$)$ y la talla promedio cérvico-caudal fue de 25 cm (D.E. 2,0 ). El diámetros céfalo-caudal promedio de los 5 riñones derechos fue de $24,4 \mathrm{~mm}$ (D.E. 2,5) e izquierdo de $24,3 \mathrm{~mm}$ (D.E. 2,8), el diámetro latero-lateral promedio derecho fue $14,3 \mathrm{~mm}$ (D.E. 0,3) e izquierdo de $15,3 \mathrm{~mm}$ (D.E.1,7) y diámetro dorso-ventral promedio derecho fue de $12,9 \mathrm{~mm}$ (D.E. 1,2) e izquierdo de 13,3 mm (D.E. 1,4).

El peso promedio de los 5 riñones derechos fue de $3,1 \mathrm{~g}$ (D.E. 0,7) e izquierdo de 3,2g (D.E. 0,9) y el volumen renal promedio (peso Scherle) de los 5 riñones, tanto derechos como izquierdos, fue de $3,1 \mathrm{ml}$ (D.E. 0,7).

La relación promedio de peso riñón derecho/peso riñón izquierdo fue de 0,96 (D.E. 0,05). La relación peso riñón/peso corporal fue de 4,5 g/Kg (D.E. 0,74).

Anatómicamente, los riñones presentan caras ventrales y dorsales; bordes mediales y laterales; y polos cefálico y caudal. Ambos riñones por su cara dorsal, a través de la cápsula adiposa, se relacionan con la pared dorsal del abdomen. En la parte media de su borde medial, se encuentra el hilio renal con sus elementos: pelvis, vena y arteria renales. En la parte cefálica del borde medial se observa la impresión de la glándula suprarrenal. Desde el borde medial y hacia dorsal los riñones se relacionan con el músculo psoas mayor.

Histológicamente, se observa a menor aumento dos zonas. La externa (corteza renal), donde se visualizan los glomérulos con sus ovillos vasculares, y entre ellos, túbulos contorneados proximales, distales, vasos sanguíneos, algunos rayos medulares y escaso intersticio de sostén. La zona interna y menos teñida (médula renal), en la que se puede observar grandes túbulos colectores y las asas de Henle más pequeñas. El intersticio de esta zona es más abundante al igual que los vasos sanguíneos.

La densidad de número $(\mathrm{Nv})$ de glomérulos de cobayo fue de $458 / \mathrm{mm}^{3}$ (D.E. $172 / \mathrm{mm}^{3}$ ) el error estándar de $74 / \mathrm{mm}^{3}$; el coeficiente de variación de $37,60 \%$ y el coeficiente de error de $16,78 \%$.

La densidad de volumen ( $\mathrm{Vv}$ ) de glomérulo de cobayo fue de 7,89\% (D.E. de 3,36\%); el error estándar de $1,5 \%$; el coeficiente de variación de $42,74 \%$ y el coeficiente de error de $19,08 \%$.

La densidad de superficie ( $\mathrm{Sv}$ ) de glomérulos cobayo fue de $3,58 \mathrm{~mm}^{2} / \mathrm{mm}^{3}$ (D.E. $1,50 \mathrm{~mm}^{2} / \mathrm{mm}^{3}$; el error estándar de $0,67 \mathrm{~mm}^{2} / \mathrm{mm}^{3}$; el coeficiente de variación de $42,15 \%$ y el coeficiente de error de $18,77 \%$.

El promedio de glomérulo del riñón de cobayo fue de 140.298 (D.E. $\pm 34.351,6$ ). Para el riñón derecho fue de 142.098 glomérulos (D.E. $\pm 26.848,0$ ) y para el riñón izquierdo fue de 138.852 glomérulos (D.E. $\pm 42.742,6$ ) por riñón.

El volumen promedio de glomérulos del riñón de cobayo fue de $244,6 \mathrm{~mm}^{3}$ (D.E. $\pm 70,75$ ). Para el riñón derecho fue de $225,0 \mathrm{~mm}^{3}$ (D.E. $\pm 51,99$ ) y para el riñón izquierdo fue de $264,4 \mathrm{~mm}^{3}$ (D.E. $\pm 91,76$ ).

La superficie promedio de glomérulos del riñón de cobayo fue de $11.086,8 \mathrm{~mm}^{2}$ (D.E. $\pm 3.343,7$ ). Para el riñón derecho fue de $10.962,4 \mathrm{~mm}^{2}$ (D.E. $\left.\pm 2.762,4\right) \mathrm{y}$ para el riñón izquierdo fue de 11.210,6 $\mathrm{mm}^{2}$ (D.E. \pm $3.950,8)$.

El volumen glomerular promedio obtenido para el cobayo, calculado por $\mathrm{Vv} / \mathrm{Nv}$ fue de $1,73 \times 10-4 \mathrm{~mm}^{3}$ (D.E. $\pm 0,11 \times 10^{-4}$ ). Para el riñón derecho fue de $1,57 \mathrm{x}$ $10-4 \mathrm{~mm}^{3}$ (D.E. $\pm 0,09 \times 10^{-4}$ ) y para el riñón izquierdo fue de $1,89 \times 10-4 \mathrm{~mm}^{3}$ (D.E. $\pm 0,19 \times 10^{-4}$ ).

El diámetro medio glomerular en el cobayo correspondió a $90 \mu \mathrm{m}$ con una desviación estándar de $\pm 9,32$, un error estándar de 2,08, un coeficiente de variación de $10,35 \%$ y un coeficiente de error de $2,32 \%$.

El glomérulo presentó un diámetro mínimo de 70 $\mu \mathrm{m}$ y un máximo de $105 \mu \mathrm{m}$. 


\section{DISCUSIÓN}

La estereología es un método de investigación que permite medir parámetros anatómicos cuantificables, confiables, reproducibles y significativos, no sólo en estructuras que presentan tejidos isotrópicos, si no también en aquellos que no lo son (Mandarim-de-Lacerda, 1995). El riñón, es un órgano no homogéneo que presenta dos zonas claramente diferenciadas, la corteza y la médula renal, siendo esta característica también propia del riñón de cobayo (Cavia porcellus).

En cuanto a forma, el riñón de cobayo es bastante similar al de otras especies, incluso al del Hombre (Frandson \& Spurgeon y Rourvière \& Delmas, 2002)

La relación peso riñón/peso corporal del cobayo fue de 4,50 g/kg, inferior si se compara con los valores obtenidos en equino y vacuno por Sisson (1982) y superior al observado en porcinos, por estos mismos autores, y en conejos (Peña et al., 2006).

Si comparamos el número de glomérulos por riñón con el informado de otras especies, es posible observar diferencias significativas entre unos y otros. Siendo menor en ratas y ratones según lo observado por Moore (1929), Bertram et al., (1992), Cullen-McEwen et al., (2001) y (2003) y Jones et al., (2001), en recién nacidos Mañalich et al., y Bassan et al., (2000) en un estudio con conejos.

Un valor mayor, aunque más cercano a este estudio, fue encontrado en conejos (Oryctolagus cuniculus) por Peña et al., y un valor más cercano pero inferior en mono verde africano (Cercopithecus aethiops) por Skov et al., (1999) y en guinea pig de 90 días (Bonvalet et al., (1977). Estos últimos investigadores utilizaron una técnica más engorrosa para la determinación de los diferentes parámetros estereológicos, ya que incluía una etapa de maceración de los riñones.

Por otro lado, un número de glomérulos por riñón mayor al del cobayo (Cavia porcellus), fue informado en perros (Omis Beagle Kennels) por Hoster et al., (1971), en oveja Border Leicester X Merino por Brown et al., (2002) y en cerdas Danesas Ladrance por Eskild-Jensen et al., (2002). Además, valores mucho mayores fueron encontrados por Hughson et al., (2003) y Hoy et al., (2003) en estudios multirraciales llevados a cabo en E.E.U.U. y Australia con muestras obtenidas de autopsias de individuos afroamericanos, aborígenes australianos y caucásicos
El volumen glomerular promedio fue de $1,73 \times 10$ ${ }^{4} \mathrm{~mm}^{3}$, parámetro que también fue muy variado según la especie consultada, así fue superior al encontrado por Mañalich et al., en humanos recién nacidos, por Armitage et al., (2005) en ratas Spague-dawley y por Roman et al., (2004) en ratas Winstar (Rattus norvergicus albinus). Sin embargo, se detectó un volumen glomerular menor en nuestro estudio, al señalado en otras especies encontradas en la literatura.

Mandarim-de-Lacerda (1995) y (2000) caracteriza la relación entre número de glomérulos y su volumen, en el riñón humano, observando un aumento del volumen glomerular y una disminución del número de glomérulos con la edad.

Los resultados obtenidos en el cobayo (Cavia porcellus) como densidad de número $(\mathrm{Nv})$, densidad de volumen ( $\mathrm{Vv}$ ) y densidad de superficie (Sv) fueron mayores que los reportados en fetos humanos y conejos.

Existe gran diferencia entre el $\mathrm{Nv}$ de conejo (Oryctolagus cuniculus) y cobayo (Cavia porcellus), siendo este último mucho mayor. Sin embargo, el número total de glomérulos por riñón es similar en ambas especies. La diferencia la presenta un tamaño renal mayor en el conejo, por lo cual, sería un factor determinante en este parámetro.

Por otro lado, también es importante considerar la zona del parénquima renal que se desea cuantificar. Así, en monos verdes africanos (Cercopithecus aethiops) se encontraron un mayor número de glomérulos en la zona superficial, pero de menor volumen, e inversamente en la zona yuxtamedular el volumen de los glomérulos era mayor, pero con un menor recuento de ellos (Skov et al.). Una dispersión similar se encontró durante el estudio histológico y una gran variedad de tamaño y número de glomérulos por campo en el estudio estereológico.

Al compara los resultados obtenidos en este estudio realizado en el riñón del cuye (Cavia porcellus) con los estudios encontrados en la literatura, es posible señalar, que factores como la especie, su edad, peso corporal, peso y volumen renal, constituyen factores preponderantes que diferencian los resultados en investigaciones morfofuncionales. Así, estas variables se deben tener presentes antes de iniciar estudios que involucren cambios morfo y cuantitativos de los riñones. 
ZAPATA, B. J. L.; DEL SOL, M. \& VÁSQUEZ, B. Renal stereology in the guinea pig. Int. J. Morphol., 27(2):419-424, 2009.

SUMMARY: The guinea pig, (Cavia porcellus) is a rodent pertaining to the Rodentia group and the Caviidae family, used as a laboratory animal and for human consumption. Quantitative parameters of the kidney provides important information of its morphofunction, given its labor in the organism's homeostasis. The aim or this study was to describe the kidney of the guinea pig (Cavia porcellus), analyzing the stereological characteristics for future experimental studies. Five male guinea pigs (Cavia porcellus) obtained from the Biotery of the Universidad de la Frontera, Temuco, Chile, were used. The kidney of the guinea pig weighed approximately $3.2 \mathrm{~g}$. The kidney has 140,298 total glomerulus, $\mathrm{Nv}$ of $458 \mathrm{~mm}^{3}$, Vv of $7.89 \%$ and $\mathrm{Sv}$ of $3.58 \mathrm{~mm}^{2} / \mathrm{mm}^{3}$. The glomerular volume of the kidney was of $1.73 \times 10^{-4} \mathrm{~mm}^{3}$ and a glomerular diameter of $90 \mu \mathrm{m}$. Factors such as species, age, body weight and renal volume, are important to consider, as they differentiate the results in the morphofunctional investigations.

KEY WORDS: Kidney; Cavia porcellus; Stereology.

\section{REFERENCIAS BIBLIOGRÁFICAS}

Bassan, H.; Laider, L.; Kariv, N.; Bassan, M.; Berger, E.; Fattal, A.; Gozes, I. \& Harel, S. Experimental intrauterine growth retardation alters renal development. Pediatr. Nephrol., 15:192-5, 2000.

Bertram, J. F.; Soosaipillai, M. C.; Ricardo, S. D. \& Ryan, G. B. Total numbers of glomeruli and individual glomerular cell types in the normal rat kidney. Cell Tissue Res., 270(1):37-45, 1992.

Bonvalet, J. P; Champion, M; Courtalon, A.; Farman, N.; Vandewalle, A \& Wanstok, F. Number of glomeruli in normal and hipertrophied kidneys of mice and guinea pigs. J. Physiol., 269: 627-41, 1977.

Brown, J.; Briscoe, T. A.; Harding, R.; Cock, M.; Bertram, J. F. \& Black, J. M. Glomerular number and capillary dimensions in the normal lamb kidney. Image Anal Stereol., 21:157-64, 2002.

Cullen-McEwen, L. A.; Drago, J. \& Bertram, J. F. Nefhron endowment in glial cell line-derived neurotrophic factor (GDNF) heterozygous mice. Kidney International., 60: 31-6, 2001.

Cullen-McEwen, L. A.; Kett, M. M.; Dowling, J.; Anderson, W. P. \& Bertram, J. F. Nethron Number, Renal function, and arterial pressure in aged GDNF heterozygous mice. Hipertension., 41: 335-40, 2003.

Eskild-Jensen, A.; Frokiaer, J. ; Djurhuus, J. C.; Jorgensen, T. M. \& Nyengaard, J. R. Reduced number of glomeruli in kidneys with neonetally induced partil ureteropelvic obstruction in pigs. J. Urol., 167:1435-9, 2002.

Fawcett, D. W. Sistema urinario. Tratado de Histología. Interamericana-Mc. Gram Hill. 1995.
Frandson, R. D. \& Spurgeon, T. L. Sistema urinario. Anatomía y fisiología de los animales domésticos. México, Interamericana-Mc. Gram Hill, 1995. pp 370-3.

Henrikson, C. Sistema urinario. Dieter, H. Histología veterinaria. Zaragoza, Acribia, 1993. pp. 233-43.

Horster, M.; Kemler, B. J. \& Valtin, H. Intracortical distribution of number and volume of glomeruli during postnatal maturation in the dog. J. Clin. Invest., 50(4):796-800, 1971.

Hoy, W. E.; Douglas-Denton, R. N.; Hughson, M. D.; Cass, A.; Jonson, K. \& Bertram, J. F. A stereological study of glomerular number and volumen: Preliminary findings in a multiracial study of kidneys at autopsy. Kidney int., 63:531-7, 2003.

Jones, S. E.; Nyengaard, J. R.; Flvbjerg, A.; Bilous, R. W. \& Marshall, S. M. Birth weight has no influence on glomerular number and volumen. Pediatr. Nephrol., 16: 340-5, 2001.

Linnaeus, C. Mus. Sistema naturae. Estocolmo, Salvius publ., 1758; 59. V. I.

Mandarim-de-Lacerda, C. A. Métodos quantitativos em Morfologia. Rio de Janiero, Ed. UERJ, 1995.

Mandarim-de-Lacerda, C. A. Fisiologia e Fisiopatologia : Estereologia do rim: determinação do Vv, Nv e volume médio do glomérulo. J. Bras. Nefrol., 22(2):103-9, 2000.

Mañalich, R.; Reyes, L.; Herrera, M.; Melendi, C. \& Fundora, I. Relationship between weight at birth and the number and size of renal glomeruli in humans: a histomorphometric study. Kidney Int., 58(2):770-3, 2000. 
Martin, R.; Marín, P. \& González, J. Roedores. Atlas de anatomía de animales exóticos. Barcelona, Masson, 2004. pp. 19-21.

Mattfeld, T.; Mall, G.; Gharehbaghi, H. \& Moller, P. Estimation of surface area and length with the Orientator. J. Microsc., 159 (3):301-17, 1990.

Moore, R. A. Number of glomeruli in kidney of adult white rat unilaterally nephrectomized in early life. J. Exp. Med. 50:709-12, 1929.

Nyengaard, J. R. Stereologic methods and their application in kidney research. J. Am. Soc. Nephorol., 10:1100-23, 1999.

Peña, E.; Romero, I.; Vásquez, B. \& Del sol, M. Determinación de parámetros estereológicos en el riñón de conejo (Oryctolagus cuniculus). Int. J. Morphol., 24(3): 331-4, 2006.

Roman, T. R. N.; de Lima, E. G.; Azoubel, R. \& Batigália, F. Renal morphometry of fetuses rats treated with cadmium. Int. J. Morphol., 22(3):231-6, 2004.

Rouvière, H. \& Delmas. Aparato urinario. Anatomía humana. Descriptiva, topográfica y funcional. Tronco: Barcelona: Ediciones Masson, 2002. V. II. pp. 465-85.

Scherle, W. A simple method for volumetry of organs in quantitative stereology. Mikroskopie, 26:57-63, 1970.

Sisson, S. Sistema urogenital de los equinos. Sisson, S. \& Grossman, J. D. Anatomía de los animales domésticos. Barcelona, Salvat Editores, 1982. V. 1. 585-90.

Skov, K.; Nyengaard, J. R.; Patwardan, A. \& Mulvany, M. J. Large juxtamedullary glomeruli and afferent arterioles in healthy primates. Kidney International, 55:1462-9, 1999.

Spotorno, A. E.; Valladares, J. P.; Marin, J. C. \& Zeballos, C. Molecular diversity among domestic guinea-pig (Cavia porcellus) and their close phylogenetic relatioships wiht the andean wild species Cavia tschudii. Revista Chilena de Historia Natural.,77:243-50, 2004.

Sterio, D. C. The unbiased estimation of number and sizes of arbitrary particles using the disector. J. Microsc., 134:127-36, 1984.

Weibel, E. R.; Kistler, G.S.; Scherle, W.F. Practical stereological methods for morphometric cytology. J. Cel. Biol., 30(1):23-38, 1966.
Dirección para correspondencia:

Prof. Dr. Mariano del Sol

Facultad de Medicina

Universidad de La Frontera

Casilla 54-D

Temuco - CHILE

Email:mdelsol@ufro.cl

Recibido : 11-01-2009

Aceptado: 22-03-2009 